\title{
COMPARISON BETWEEN NORMAL AND HEII TWO-PHASE FLOWS AT HIGH VAPOR VELOCITIES
}

\author{
S. Perraud ${ }^{1,2}$, B. Rousset ${ }^{1}$, P. Thibault ${ }^{2}$, R. van Weelderen ${ }^{3}$, P. E. Wolf ${ }^{2}$
}

We present results on helium co-current two-phase flow experiments at high vapor velocity obtained with the use of the new CEA/SBT $400 \mathrm{~W} / 1.8 \mathrm{~K}$ refrigerator [1]. For vapor velocities larger than typically $4 \mathrm{~m} / \mathrm{s}$, a mist of droplets develops from the bulk liquid interface accompanied by an increase in heat transfer at the wall. Experiments were conducted in a $10 \mathrm{~m}$ long, $40 \mathrm{~mm}$ I.D. straight pipe, both in helium II and in helium I to compare these two situations. The respective roles of vapor density, vapor velocity and liquid level on atomization were systematically investigated. Light scattering experiments were performed to measure sizes, velocities and interfacial areas of droplets in a complete cross section. In-house-made heat transfer sensors located in the mist allowed us to deduce an upper value of the extra cooling power of the dispersed phase. The practical interest of atomized flow for cooling large cryogenic facilities is discussed by considering the balance between increase in heat transfer and pressure drops it induces.

1 DRFMC/SBT/CEAG, Grenoble, France

2 CRTBT/CNRS, Grenoble, France

3 CERN, Accelerator Technology Department, Geneva, Switzerland

Presented at the CEC-ICMC'05 Conference

29 August-2 September 2005, Keystone, Colorado, USA

Administrative Secretariat

AT Department

CERN

CH - 1211 Geneva 23
Geneva, Switzerland

19 January 2006 
COMPARISON BETWEEN NORMAL AND HEII TWO-PHASE FLOWS AT HIGH VAPOR VELOCITIES

\author{
S. Perraud ${ }^{1 / 2}$, B. Rousset ${ }^{1}$, P. Thibault ${ }^{2}$, R. van Weelderen ${ }^{3}$, P. E. Wolf $^{2}$ \\ ${ }^{1}$ DRFMC/SBT/CEAG \\ Grenoble, 38054, France \\ ${ }^{2}$ CRTBT/CNRS \\ Grenoble, 38042, France \\ ${ }^{3}$ CERN, European Organization for Nuclear Research, AT/CR, CH-1211 \\ Geneva, 23, Switzerland
}

\begin{abstract}
We present results on helium co-current two-phase flow experiments at high vapor velocity obtained with the use of the new CEA/SBT $400 \mathrm{~W} / 1.8 \mathrm{~K}$ refrigerator [1]. For vapor velocities larger than typically $4 \mathrm{~m} / \mathrm{s}$, a mist of droplets develops from the bulk liquid interface accompanied by an increase in heat transfer at the wall. Experiments were conducted in a $10 \mathrm{~m}$ long, $40 \mathrm{~mm}$ I.D. straight pipe, both in helium II and in helium I to compare these two situations. The respective roles of vapor density, vapor velocity and liquid level on atomization were systematically investigated. Light scattering experiments were performed to measure sizes, velocities and interfacial areas of droplets in a complete cross section. In-house-made heat transfer sensors located in the mist allowed us to deduce an upper value of the extra cooling power of the dispersed phase. The practical interest of atomized flow for cooling large cryogenic facilities is discussed by considering the balance between increase in heat transfer and pressure drops it induces.
\end{abstract}

KEYWORDS: Two-phase flow, sprays, heat transfer, He II

PACS: 07.20.Mc 


\section{INTRODUCTION}

In the CERN Large Hadron Collider, the superconducting magnets will be cooled by a He II two-phase flow. The purpose of the Cryoloop experiment [2] was to study the heat transfer capabilities provided by such a flow. We previously showed that, for vapor velocities exceeding typically $4 \mathrm{~m} / \mathrm{s}$, the heat transfer between the wall of the pipe and the HeII two-phase flow is improved with respect to a purely stratified situation [3]. Optical observations showed that this improvement is correlated to the appearance of a droplet mist inside the pipe. We assumed that the deposition of these droplets creates a thin liquid film on the walls. The increase of the wetted surface enhances the wall heat transfer. In this paper, we confirm this mechanism by measuring locally the improvement of the heat transfer, using suspended heaters (thermal 'flags') located inside the mist [4]. We also take benefit of the capabilities of our new refrigerator to explore a wider range of conditions, in order to answer the following issues: Is there a limit in the heat transfer improvement when the vapor velocity increases or could the wetted perimeter reach $100 \%$ ? Does this heat transfer improvement still exist in normal helium?

\section{EXPERIMENTAL FACILITY INSTRUMENTATION AND METHODS}

\section{Experimental facility}

Our new large refrigerator marked I on FIGURE 1 is presented elsewhere [1]. It is able to absorb a variable heat load (maximum of $400 \mathrm{~W}$ ) at a temperature ranging between 1.8 and $4.4 \mathrm{~K}$. It delivers liquid helium at $T_{\lambda}$ and 1 bar upstream at the two Joule-Thomson valves (VJT1 and VJT2). Valve VJT1 controls the mass flowrate delivered to the test line, while valve VJT2 is used to control the liquid level of the sub-cooler bath NS2.

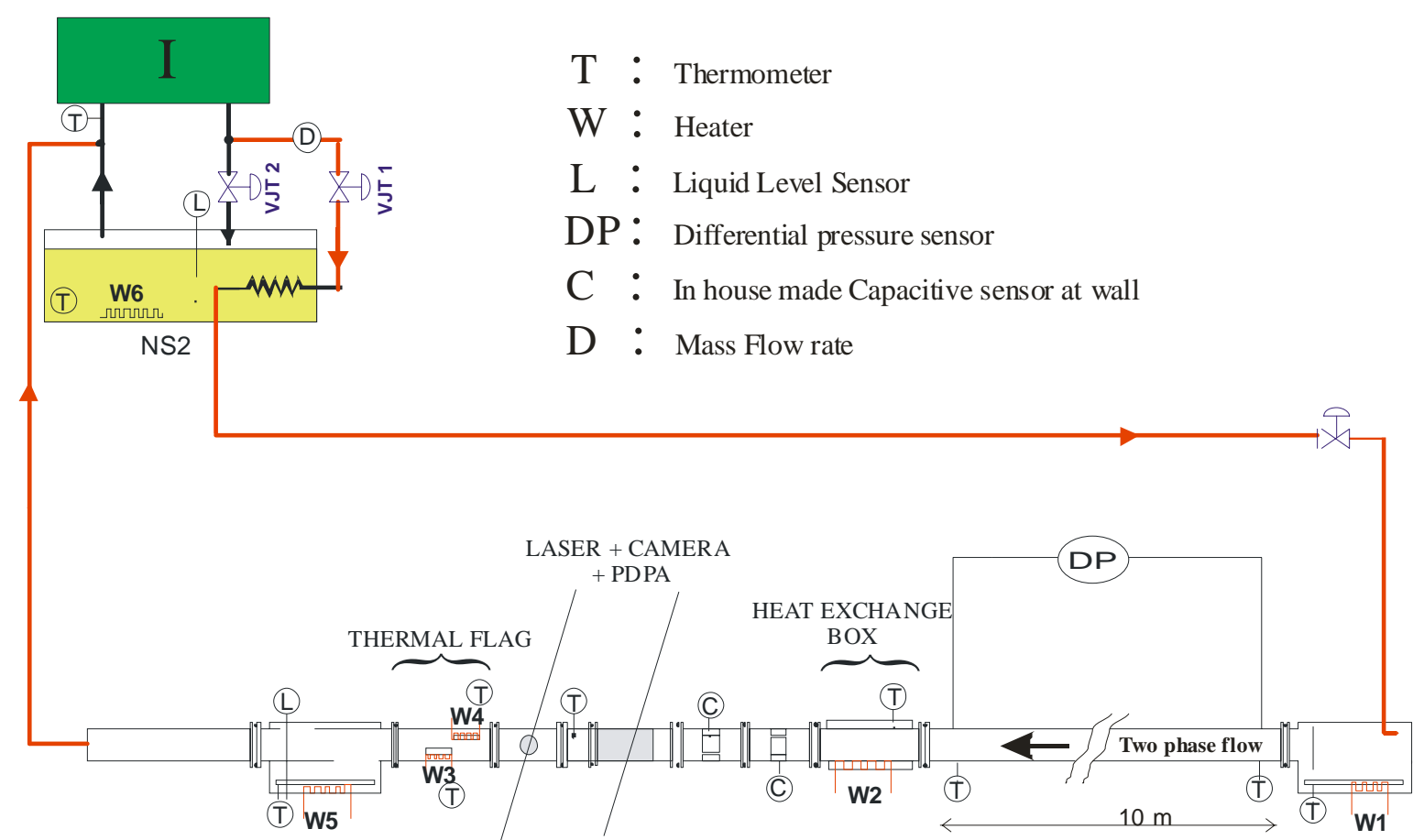

FIGURE 1. Process and instrumentation diagram of the helium two-phase flow facility 
The NS2 temperature is selected by adapting its pressure by means of two centrifugal cryogenic compressors located inside I. From upstream to downstream, the various elements composing the test line are: the valve VJT1 used to control the required mass flow (measured upstream of this valve using a cryogenic Coriolis mass flowmeter), a subcooler heat exchanger made up of a $16 \mathrm{~m}$ long aluminum coil immersed in the outlet saturated bath and used to obtain at its outlet a temperature close to that of the saturated bath, at a pressure of about $10 \mathrm{KPa}$, a final pressure discharger, an inlet box in which is located a heater $(<400 \mathrm{~W})$ which is used to impose the vapor mass flow, the two-phase flow line (inner diameter $40 \mathrm{~mm}$ - $13 \mathrm{~m}$ long) instrumented on its last 3 meters, a phase separator allowing to evaporate the excess of liquid flowing at the bottom of the tube and, finally, connections to the pumping line of the $400 \mathrm{~W} / 1.8 \mathrm{~K}$ refrigerator.

\section{Instrumentation of the test line}

In the same way, from upstream to downstream, we measure the mass flow (Coriolis mass flowmeter), the power used to create the vapor flow, the temperature at both ends of the line and the corresponding differential pressure, the heat transfer through a heat exchange box [2] filled with 1 bar pressurized helium which is converted into an equivalent wetted perimeter $(\mathrm{P})$, and the level of bulk liquid by means of parietal capacitive sensors azimuthally distributed [4]. Two visualization zones are also used to characterize the droplet flow (measurement of interfacial area, velocity and diameter of droplets [5]). Thermal "flags" [6] located inside the vapor flow are used to characterize the droplet cooling power. Each 'flag' consists in a plane heater parallel to the flow (one side of which is thermally insulated by a plastic piece and the other is in contact with helium) and a thermometer measuring the plate temperature as a function of applied power. Other measured quantities are the power controlling the phase separator level and, finally, the temperature and the power injected and the liquid level of the sub-cooler bath.

\section{Experimental procedure}

The use of two parallel circuits to feed on the one hand the sub-cooler bath containing the subcooler and on the other hand the test line makes it possible to vary quality from 0 to 1 in the line for temperatures higher than $1.75 \mathrm{~K}$ and for a total mass flow rate (liquid + vapor) up to $20 \mathrm{~g} / \mathrm{s}$. Since this study is dedicated to the behavior at high vapor velocities, only mass flow rates larger than $7 \mathrm{~g} / \mathrm{s}$ were explored. These large vapor velocities shear the liquid free surface, producing droplets which are dragged by the vapor flow before settling on the wall. This phenomenon appears for critical vapor velocities close to $4 \mathrm{~m} / \mathrm{s}$.

Although the inception criteria for partially atomized flow certainly depends on the formation of waves (related to Kelvin-Helmholtz instabilities), one expects that the final droplet size corresponds to some critical value of the Weber number $\left(W e=\rho_{g} \mathrm{v}_{g}{ }^{2} d_{d} / \sigma\right.$, where $\rho_{g}, \mathrm{v}_{g}, d_{d}$ and $\sigma$ are respectively density and velocity of vapor, droplet diameter and surface tension). This expresses the competition between the droplet shearing forces ( $\rho_{\mathrm{g}}$ $\mathrm{v}_{\mathrm{g}}{ }^{2}$ ) and the Laplace forces $\left(\sigma / d_{d}\right)$ responsible for the droplet cohesion.

In order to determine the influence of the various parameters likely to influence the characteristics of the atomization (inception, size and density of droplets, height of stratification), we varied independently the density and the velocity of the vapor, as well as the liquid level in the tube. For two-phase flows, the vapor density depends only on its temperature. Temperatures ranging between 1.8 and $2 \mathrm{~K}$ and also a temperature of $2.33 \mathrm{~K}$ (normal fluid) were explored, corresponding to a factor of 4 in the change of density. The 
desired value of the temperature is fixed by the refrigerator (I) by adapting the speed of the cold compressors (and by throttling the flow thanks to a valve located upstream of the cold compressors if necessary).

For a given superficial vapor velocity $\mathrm{U}_{\mathrm{GS}}$, the power $\mathrm{W} 1$ to be applied to the inlet heater is computed as $W 1=\rho_{\text {vap }} S_{\text {tot }} U_{G S} L_{\text {sat }}-W_{\text {losses }}$ with $\rho_{\text {vap }}$ the vapor density, $S_{\text {tot }}$ the whole cross section area, $U_{G S}$ the superficial vapor velocity, $L_{\text {sat }}$ the latent heat of vaporization and $W_{\text {losses }}$ the heat losses along the line. The actual velocity on the pipe axis is then measured with a Phase Doppler Particle Analyzer and tuned to the desired value by adjusting $\mathrm{W} 1$.

The total mass flow is adjusted to reach the desired liquid level, as measured by the parietal capacitive probes. Liquid level and vapor velocity being only slightly coupled, and the void fraction being always important, this adjustment of the working point is easily obtained.

\section{RESULTS AND ANALYSIS}

\section{Influence of Vapor Velocity}

The vapor velocity reasonably ought to be the main parameter governing the rate of atomization. We have checked its influence for a given liquid level $h$, so as to have a fixed surface of atomization ( $h / D=8 \%$ and $l / D=55 \%$, as defined in FIGURE 3b).

FIGURE 2a represents $\Sigma$, the total liquid droplet interfacial area per unit of volume measured using light scattering [5], as a function of the height in the pipe $(y / D=0$ at the bottom). It shows that the mist is stratified (the maximum corresponds to the liquid vapor interface) and becomes denser as the velocity is increased. FIGURE 2b (right scale) shows that the total wetted perimeter $(P)$ obtained from heat exchange measurements, also increases with the vapor velocity. For the largest vapor velocities, it was possible to reach a heat transfer equivalent to $P$ of approximately $90 \%$. For the studied liquid level, $P$ increases roughly linearly with $U_{G S}$. In order to single out the film contribution to this increase, we subtract the constant bulk liquid contribution to $P$ (as measured by the capacitive probes) and normalize the result by its maximum value, for all vapor velocities and temperatures tested. This quantity, called $P_{\text {norm}}$, is compared to $\Sigma$ at mid-height of the tube, also normalized by its largest value, and both quantities increase with the vapor velocity, which is suggestive of the role of droplets in the formation of the film.

More direct evidence is provided by the local thermal 'flags'. In the presence of mist, as the 'flags' heating power is increased, their temperature rises first with a slope characteristic of a fully wetted sensor. Above some critical threshold $W_{\text {crit }}$, the slope increases sharply, corresponding to a burnout crisis, above which the flag dries out. If the film is formed from the mist, we expect $W_{\text {crit }}$ to be given by the incident flux of liquid droplets onto the heater, times the latent heat, hence to be proportional to $\Sigma$, i.e. to decrease with increasing height. As shown by FIGURE 2c, this is indeed the case.

Overall, FIGURES 2a to 2c confirm the large influence of the vapor velocity on the mist density and heat transfer. In contrast, the droplet Sauter mean diameter $\left(D_{32}\right)$ remains approximately $45 \mu \mathrm{m}$ in the range 10 to $17 \mathrm{~m} / \mathrm{s}$ (FIGURE 2d). These figures also show that, unexpectedly, the temperature has a small role, if any, from $1.8 \mathrm{~K}$ to $2 \mathrm{~K}$, although the vapor density changes by a factor close to 2 in this range. 


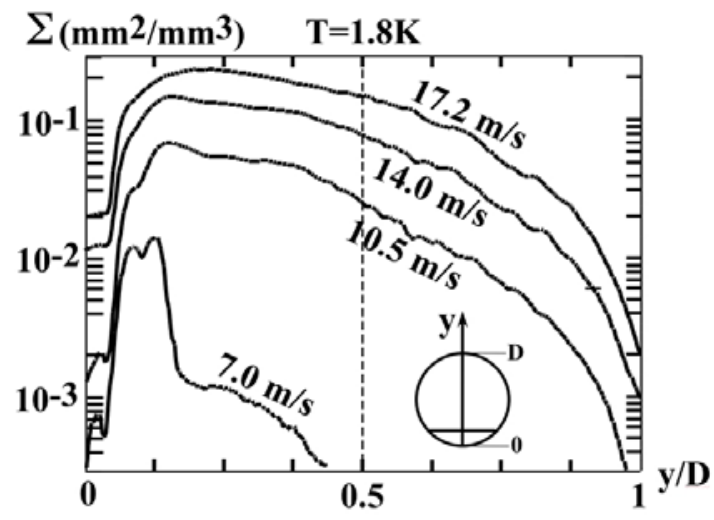

FIGURE 2a. volumic interfacial area as a function of normalized height for various superficial vapor velocities

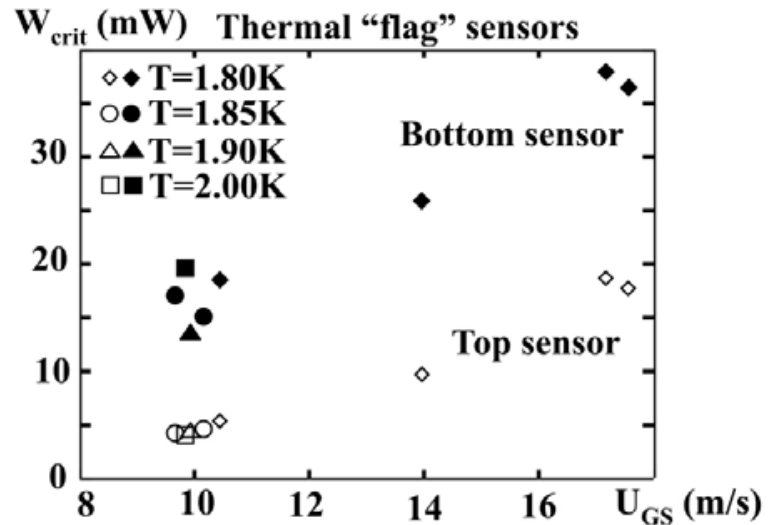

FIGURE 2c. Critical power (i.e. inception of burnout crisis) for thermal flag below and above the pipe center) as a function of superficial vapor velocity

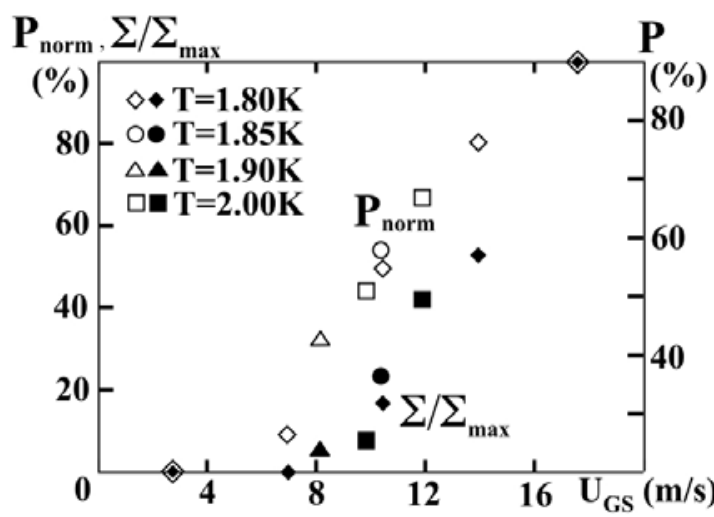

FIGURE 2b. Normalized wetted perimeter and normalized interfacial area per unit of volume as a function of superficial vapor velocity

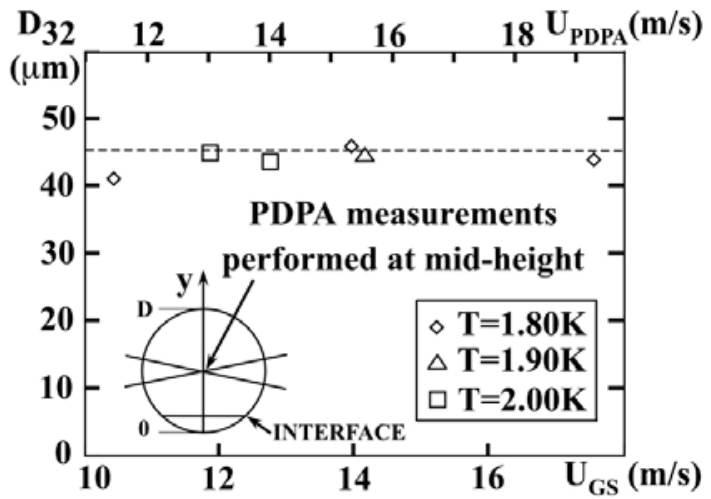

FIGURE 2d. Sauter diameter

$\left(D_{32}=\frac{\text { total volume of spray particle }}{\text { surface area of particle }}\right)$ of the liquid droplets as measured at the center of the pipe

This is confirmed by FIGURE 4a, which gives the profile of the interfacial density, for a fixed liquid level and vapor velocity, and different temperatures, as well as the critical power for the two thermal flags.

\section{Influence of Liquid Level}

Below mid-height, raising the liquid level increases the surface of atomization $/ / D$

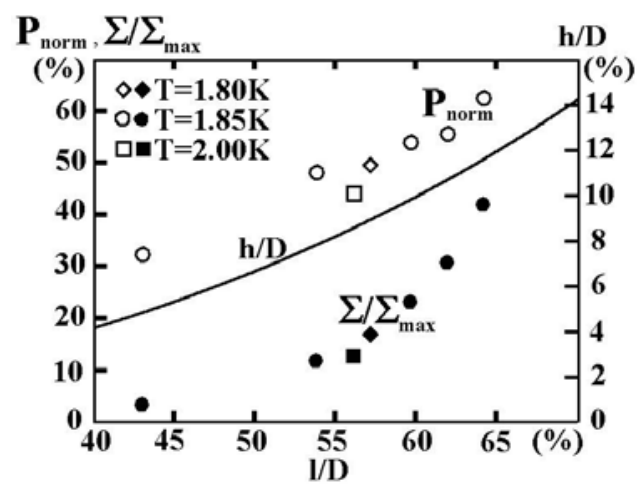

FIGURE 3a. Normalized wetted perimeter, interfacial area and height as a function of normalized length interface

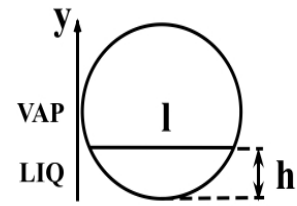

inner diameter $\mathrm{D}$ interface width 1 liquid height $h$

FIGURE $3 b$.

definition of various parameters

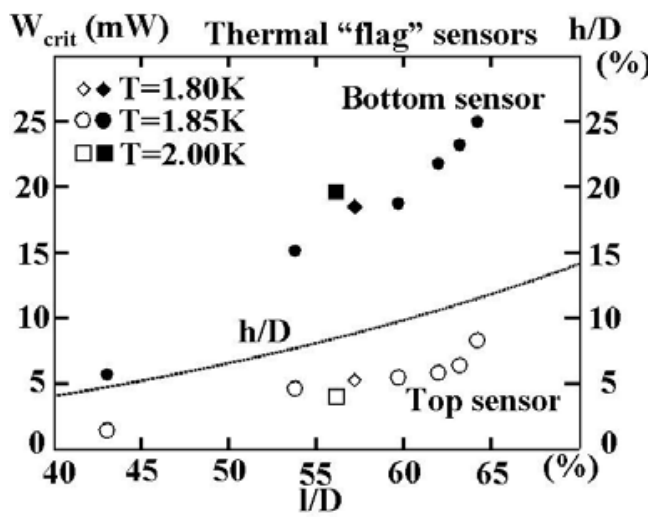

FIGURE 3c. Critical power and height as a function of normalized length interface 
(FIGURE 3b). As shown by FIGURE 3a, this results in an improved heat transfer for a given vapor velocity and vapor density. The interfacial area density at mid-height also increases, but more strongly. This might be an effect of the increase of the interface level, combined with stratification. Associated with this increase in atomization, $W_{\text {crit }}$ for the thermal 'flags' also increases (FIGURE 3b), providing a direct evidence for the role of the mist in the thermal transfer.

\section{Comparison between Normal and Superfluid Helium}

The large liquid flow rate delivered by the new refrigerator allows us to apply, without drying, the heating power necessary to reach velocities of order $10 \mathrm{~m} / \mathrm{s}$ in the normal phase. Using the number of counts recorded by the phase doppler particle analyser (PDPA) as an inception criterion for atomization, we find the critical (on-axis) velocity to be equal to $4.3 \mathrm{~m} / \mathrm{s}$ at $1.8 \mathrm{~K}$ and $3.8 \mathrm{~m} / \mathrm{s}$ at $2.33 \mathrm{~K}$. As this small difference could result from the difficulty to properly regulate the normal helium flow, atomization appears insensitive to superfluidity, which is not unexpected. Above this threshold, FIGURE 4b shows that the droplets distribution, for fixed velocity and liquid level, does not depend on superfluidity either (nor, in a similar way to FIGURE 4a, on the factor of two increase of the vapor density between $2 \mathrm{~K}$ and $2.33 \mathrm{~K}$ ).

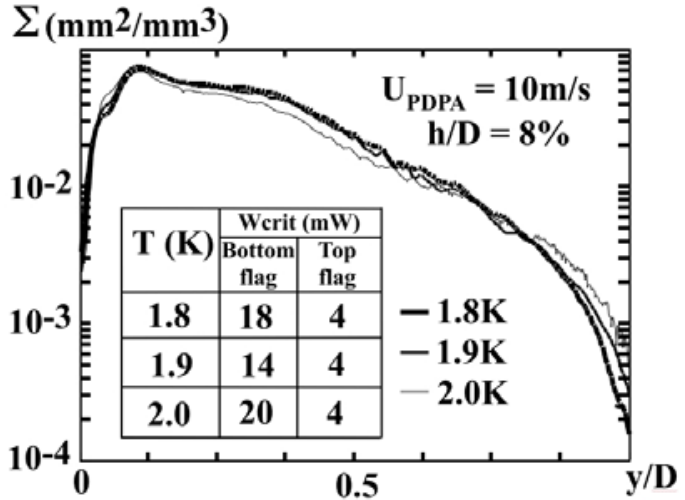

FIGURE 4a. Influence of density on atomization and thermal transfer

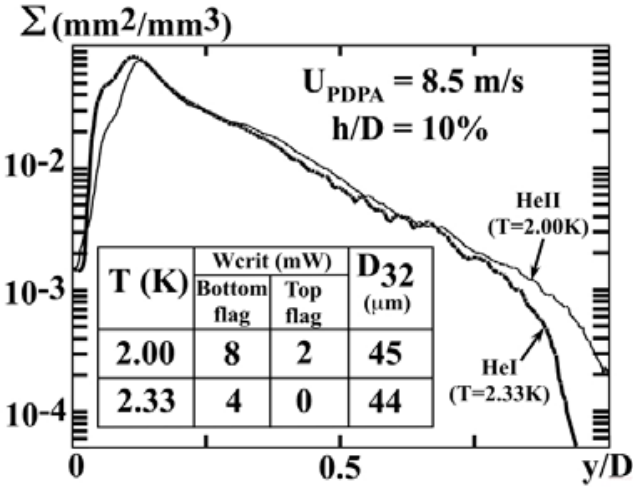

FIGURE 4b. Comparison of atomization and thermal transfer for HeI and HeII

Total mass flow $14.5 \mathrm{~g} / \mathrm{s}$, Outlet temperature $1.8 \mathrm{~K}$

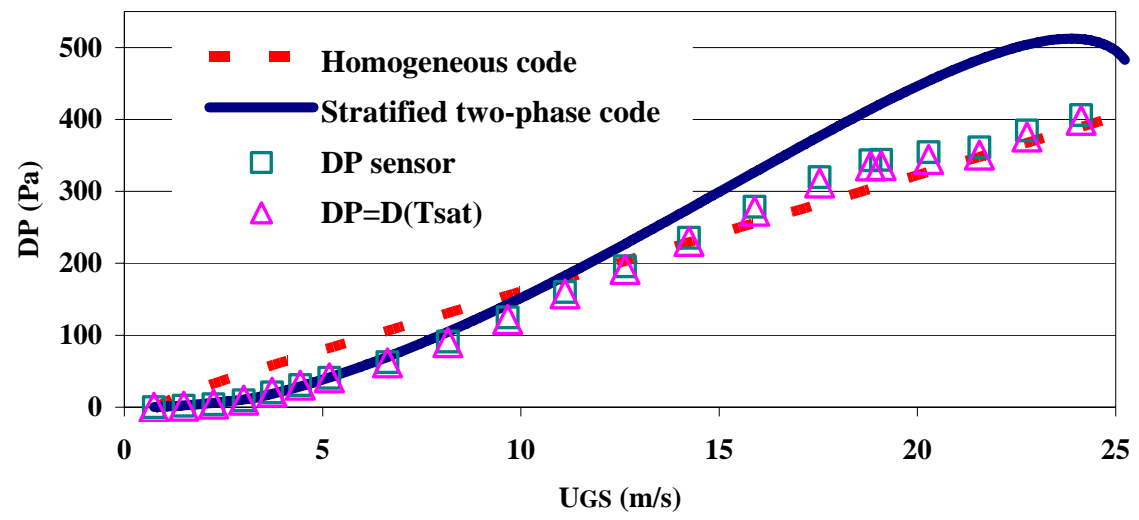

FIGURE 5. Comparison of experimental pressure losses with various models 
As shown by the inset of FIGURE 4b, although $\Sigma$ and $D_{32}$ are the same, the critical power for the thermal flags is lower in the normal phase than in the superfluid phase, dropping to zero for the top flag. However, another measurement at lower temperature ( $2.24 \mathrm{~K})$ shows a less pronounced decrease, so that the data have to be analyzed in more detail to quantify the effect of superfluidity on the cooling efficiency. A factor of two difference in $W_{\text {crit }}$ could be explained by a larger effective cross section of the sensor for droplets in the superfluid case, due to a superfluid flow between the thermally insulated (cold) face and the (warm) conductive face of the flags. Alternatively, a larger difference would need to invoke a less efficient spreading of the impinging droplets in the normal phase.

\section{Pressure Drop}

We come to the conclusion that a flow with a large fraction of entrainment has a very interesting cooling capacity, but the price to be paid in terms of pressure losses has to be known. FIGURE 5 shows a comparison between the measured and calculated two-phase flow pressure losses. When the vapor velocity is lower than $11 \mathrm{~m} / \mathrm{s}$, our previous stratified two-phase flow model [5] can be applied. At higher velocities and higher qualities, discrepancies appear and the flow behavior is more similar to a homogeneous flow.

The knowledge of this behavior and the various agreements between results and the different predictions suggest that we have a reliable tool for dimensioning. On the other hand, the importance of pressure losses at large vapor velocities restricts the practical capability to extract large linear heat losses to a rather short section of a line.

\section{DISCUSSION}

Our results show that the perimeter fraction that can be wetted by liquid helium can reach values close to $100 \%$ for the highest vapor velocities. In such a mode, the resulting pressure losses for a line of $10 \mathrm{~m}$ in length correspond to a temperature variation lower than $0.1 \mathrm{~K}$ at $1.8 \mathrm{~K}$, which may still be acceptable for some selected applications.

Under such conditions, the thermal flags give an indication of the extractable heat flux before burnout crisis at the wall. However, these indications strongly depend on the azimuthal position considered, related to the liquid droplet flow stratification.

Considering the thermal flag located above the line axis, the thermal power extracted before burn out exceeds $80 \mathrm{~W} / \mathrm{m}^{2}$ for a flow of $9 \mathrm{~g} / \mathrm{s}$ and a vapor velocity of $10.5 \mathrm{~m} / \mathrm{s}$ at a temperature of $1.8 \mathrm{~K}$. This number assumes that only the droplets impinging on the sensitive part of the flag are effective for cooling, and could be decreased by a factor of two if, due to superfluid flow, the full sensor surface contributes.

Below this limit in thermal power, at least $50 \%$ of the pipe area will be wetted (since the thermal flag considered is located above mid-height), and the temperature difference between two-phase flow and the magnet to be cooled will be minimized. It is attractive to link this cooling capacity to the mass flow of droplets which impinge on the flag.

In order to estimate this quantity, we take from our optical measurements an average diameter of $45 \mu \mathrm{m}$ ( $D_{32}$ as measured by the PDPA) and an average value of $\Sigma$ of $10^{-2}$ $\mathrm{mm}^{2} / \mathrm{mm}^{3}$ (taken at the height of the top thermal flag). Assuming further that the droplets radial velocity, $v_{r}$, is on the order of the fluctuations of the axial velocity measured by the PDPA (about $1 \mathrm{~m} / \mathrm{s}$ ) and the volume flow rate incident on the walls (per unit area) is 
$\Sigma D_{32} / 6 v_{r}=710^{-2}\left(\mathrm{~mm}^{3} / \mathrm{s}\right) / \mathrm{mm}^{2}$. Given the latent heat, this corresponds to a cooling power at the walls of $25 \mathrm{~W} / \mathrm{m}$ or $200 \mathrm{~W} / \mathrm{m}^{2}$. In order of magnitude, this value is comparable to the critical thermal power measured by the thermal flag. The factor of 3 difference could be due to a smaller impinging velocity than assumed, or to a non perfect efficiency of the deposition process (i.e. to some bouncing of the droplets). A definitive conclusion would require a direct measurement of the impinging velocity.

\section{CONCLUSION}

By using our new refrigerator, we were able to enlarge the range of vapor velocities and temperature previously studied in two-phase HeII. The increase in vapor velocities allows an improvement of the wetted surface fraction (i.e. heat exchange), with a maximum value close to $100 \%$. At the same time, pressure losses can be predicted using a previous two-phase flow model for vapor velocities below $11 \mathrm{~m} / \mathrm{s}$ and by a homogeneous model for velocities above that.

It was possible to perform some tests in the normal helium domain. In that case, the thermal "flag" sensors only were used to determine the heat transfer, as heating the pressurized normal bath would result in a large thermal gradient inside the bath, due to poor heat conductivity of normal helium. Comparative results in normal and superfluid helium show that dry out crisis seems to appear at a lower value of heat flux for HeI than for HeII, in conditions where the density and velocity of droplets are similar. A possible explanation could be that, in the superfluid case, the droplets efficient for cooling are captured over a larger area, thanks to the superfluid flow.

\section{ACKNOWLEDGEMENTS}

We would like to thank the whole CEA/SBT/GTh team for their technical help in assembling the test line and running the $400 \mathrm{Watt} / 1.8 \mathrm{~K}$ refrigerator.

\section{REFERENCES}

1. Roussel P. and al., "The $400 \mathrm{~W}$ at $1.8 \mathrm{~K}$ test facility at CEA-Grenoble" to be published in the proceeding of this conference

2. Rousset B. et al., "HeII two phase flow in an inclinable $22 \mathrm{~m}$ long line”, Advances in Cryogenics Engineering_(2000), vol 45 1009-1016

3. Rousset B. et al., "Heat Transfer Enhancement of He II Co-current Two-phase Flow in The Presence of Atomisation” International Cryogenics Engineering Conference 20 Beijing, China (2004), to be published

4. Thibault P. et al., "Probing the Wetted Perimeter in a Pipe-Flow Experiment Using a Capacitive Sensor”, Advances in Cryogenics Engineering (2002) vol 47 1683-1690

5. Wolf P.-E. et al., "PDPA Characterization of The Droplet Mist in a HeII Two-phase Flow” International Cryogenics Engineering Conference 19 Grenoble, France(2002), 667-670

6. Thibault P. et al., "Description of a new experiment to explore HeII two phase flow behavior at high mass flow rate” International Cryogenics Engineering Conference 19 Grenoble, France(2002), 825-828

7. Grimaud L. and al., "Stratified Two-phase Superfluid Helium Flow-Part: II" Cryogenics 37 (1997) 